present $20 \times 20 \times 25$-nanometre resolution limit of SBF-SEM. However, SBF-SEM can image much greater volumes - on the scale of two to three orders of magnitude more - making focused ion beam and SBF complementary rather than competitive tools.

\section{A beautiful mind}

There are alternatives to electron microscopy - particularly for researchers interested in more than a static snapshot. "All the things I've studied up until now have been dynamic questions," says Lichtman. "And you just can't do that with electron microscopy - you've got to kill it to look at it!"

Lichtman's solution was the Brainbow transgenic mouse ${ }^{3}$, which uses a site-specific DNA recombination system to randomize expression of multiple fluorescent protein genes in neurons, yielding intermediate colour combinations that distinguish each cell from its neighbours. With a broad portfolio of commercially available fluorescent proteins from which to choose - including the Living Colors proteins made by Clontech in Mountain View, California, and the TurboColors proteins from Evrogen in Moscow - Lichtman's group had many options. However, just a handful of colours proved sufficient to generate nearly 100

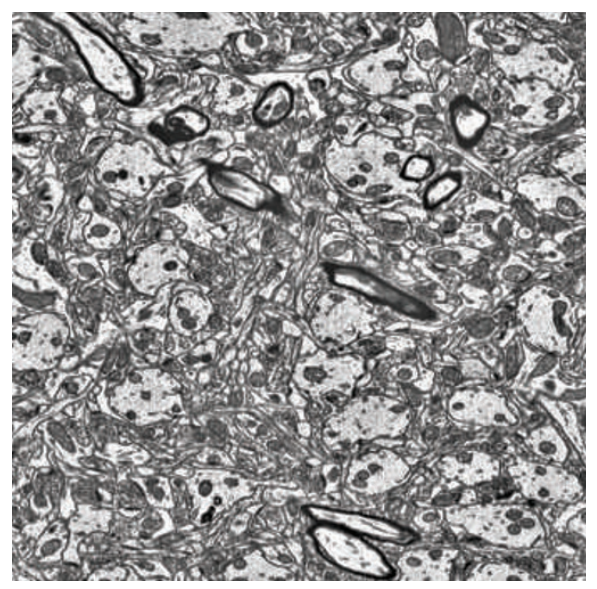

Focused-ion-beam microscopy, as performed with instruments such as FEl's Helios NanoLab DualBeam, allows more energy to be used for imaging, improving the resolution.

distinct labels. "All of the colours of the rainbow that we see are interpreted from three pigments in our retina," he explains. "So we just inverted that, thinking that if we could just mix different amounts of three colours in different cells, we should be able to get all the visible colours of the rainbow."

In other cases, more selective labelling is desirable, and scientists since Ramón y Cajal have pursued chemical and biological methods for exclusively targeting neurons that are functionally linked via active synapses. One promising method, being pioneered by scientists such as Lynn Enquist at Princeton University in New Jersey and Ed Callaway at the Salk Institute for Biological Studies in La Jolla, California, exploits natural infection patterns of neurotropic viruses for the fluorescent labelling of individual neural circuits ${ }^{4}$.

Callaway works with modified rabies virus, a pathogen that spreads so efficiently across mouse neurons that a single particle injected into the brain can prove lethal. His viruses are constrained via deletion of a key glycoprotein gene. "We preserved the ability to replicate and amplify, but provided a means to control the spread," he says. "Deleting the glycoprotein gene also allows us to control the initial infection and target specific cell types." Some investigators are applying viral tracing to trace entire networks of interconnected cells, but Callaway is mostly interested in targeting smaller 'neighbourhoods'. "When we get to the point where we can go into a live animal and target one cell and label every single input to that cell, that will be a huge advance," says Callaway. "But it's clear we're far from labelling all of them. We're now labelling

\title{
WHOSE MAP IS IT ANYWAY?
}

Even as 'connectomics' makes its way into the mainstream scientific vocabulary, there is already debate over what - if anything — it actually means. "It's sort of analogous to how 'genome' used to mean the set of genes, but now it means the whole DNA sequence," explains Sebastian Seung of the Massachusetts Institute of Technology in Cambridge.

There is fairly broad agreement that mapping the wiring in mammalian brains is a worthwhile endeavour. The issue is one of scale - should these be comprehensive reconstructions of neuronal circuitry, or more macro-scale representations of long-range connections between regions of the brain? This is the neuroanatomical equivalent of choosing between creating a road atlas or Google Earth.

Arguments can readily be marshalled for and against either approach; most boil down to how best to invest time, money and technology. "Dense reconstruction of a cubic millimetre of the cortex is kind of a 'going to the Moon' goal, where we think it's possible but difficult," says Seung, "but in solving that problem, all the other problems become trivial."

On the other hand, Partha Mitra of Cold Spring Harbor Laboratory in New York thinks that the tools are already at hand for creating a sparser 'mesoscopic' map of the projections that link functionally discrete brain regions, which some call a projectome - although you won't catch Mitra using that term. "Everything has an '-ome' added to it, and that's ok if you're in a yoga class," he jokes. "But I prefer 'brain architecture' because it conveys structure and function; architects shape space for human use, and evolution shaped our nervous system for appropriate behavioural repertoires and so on."

Mitra and dozens of colleagues recently published a plan for integrating existing tools including chemical labels and engineered viral tracers - into a concerted effort to chart the connections between functionally homogeneous clusters of cells via light microscopy ${ }^{9}$. Larry Swanson at the University of Southern California, Los Angeles, has proposed that 500-1,000 such anatomical units exist within the brain, and Mitra thinks that linking these will prove challenging but not insurmountable. "Larry has deep knowledge of the relevant literature, and estimates that only around a third of these possible mesoscopic connections have ever been studied," Mitra says. "But when I sat down and thought about the cost to map out those connections, I was shocked to find that it actually shouldn't take that much time, money or effort." With the recent awarding of a Transformative R01 grant from the US National Institutes of Health, Mitra's team is now taking first steps towards making their Mouse Brain Architecture Project into a reality.

At the same time, by deliberately overlooking the highest orders of neuroanatomical complexity, this approach leaves open numerous questions that will probably be answered only by dense mapping. "These things are just so incredibly tangled and complicated, it's inconceivable that you'll come across two identical brains," says Stephen Smith of Stanford University in California. "And I think it is a wonderful opportunity for those of us who are pursuing connectomes - to do not one, but many. Neural plasticity is just one of the many interesting questions that will be open for new attack."

Scientists in both camps hold up work done by researchers at the Allen Institute for Brain Science in Seattle, Washington, in mapping gene expression in the brain as an example of how good science, careful planning and efficient workflows can yield tremendous pay-offs. Smith and others think that maturation of high-resolution circuit-mapping techniques will ultimately bring high-throughput 'dense' reconstruction within reach.

Accordingly, Mitra emphasizes that his group's data - which it intends to make freely available via an open-access model should provide a framework for future reconstructions. "This is just supposed to be the first generation," he says. "I have no doubt that if this succeeds - this whole-brain approach to brain architecture and neuroanatomy - then we will see successive waves of technology hitting the problem." 